Bundesgesundheitsbl -

Gesundheitsforsch - Gesundheitsschutz

2000 • 43:346-350 @ Springer-Verlag 2000

Leitthema: Umwelt und Gesundheit

U. Kaiser

Robert Koch-Institut, Berlin

\title{
Auf- und Ausbau einer euro- päischen Informations- und Kommunikationsplattform
}

\section{Zusammenfassung}

Der Bereich „Umwelt und Gesundheit” zeichnet sich durch einen interdisziplinären Charakter und einen daraus resultierenden hohen Kommunikationsbedarf aus. Erschwerend kommt hinzu, dass sich mitunter bei der Informationsbeschaffung erhebliche Probleme ergeben. Ein Vergleich nationaler Problemlösungsstrategien in Form elektronischer Vernetzungsaktivitäten auf diesem Gebiet hat zum Entwicklungsprojekt für eine europäische Informations- und Kommunikationsplattform für diesen Bereich geführt. Internationaler Erfahrungsaustausch, koordinierte Vorgehensweisen, Harmonisierungsbestrebungen und Qualifizierungsmöglichkeiten für ,environmental health professionals" sollen hierdurch erleichtert werden.

\section{Schlüsselwörter}

Umwelt und Gesundheit · elektronische Vernetzung · europäische Informations- und Kommunikationsplattform
E in großes Problem für die mit Fragen aus dem Bereich „Umwelt und Gesundheit" beschäftigten Ärzte, Naturwissenschaftler, Verwaltungsbeamten, Ministerien und sonstigen Behörden besteht in der Informationsbeschaffung, da die an sie herangetragenen Probleme oft in Bereichen liegen, die nicht in gängigen Lehrbüchern, Nachschlagewerken etc. zu finden sind. Im Gegensatz zur „etablierten Medizin” gibt es in der „Umweltmedizin" noch keinen über Jahrhunderte gewachsenen Wissensstand, und folglich ist das Angebot an speziellen „Umweltmedizin-Fachbüchern” noch an zwei Händen abzuzählen. Recherchen in internationalen Faktenoder Literaturdatenbanken liefern zu subtilen Fragestellungen mitunter keine adäquaten Antworten oder stellen den Anfragenden vor neue Probleme, wenn es sich beispielsweise trotz zahlreicher vorhandenen „Treffer” bei der Recherche um widersprüchliche Informationen handelt oder um Informationen, deren Interpretation einen großen Spielraum zulässt bzw. detailliertes Fachwissen (z.B. Risikoabschätzungen von Kombinationswirkungen, klinische Relevanz immuntoxikologischer Parameter etc.) erfordert.

Erschwerend kommt hinzu, dass in der Öffentlichkeit immer häufiger Umweltnoxen (biologische, chemische, physikalische Faktoren) in kausalem Bezug zu subjektiven Beschwerden bzw. medizinisch manifesten Befunden gesehen und von den Massenmedien mitunter in spektakulärer Weise thematisiert werden. Der Stellenwert einer kompetenten Risikokommunikation wird inzwischen von niemanden mehr angezweifelt.

\section{„Immer häufiger werden \\ Umweltnoxen in kausalem Bezug zu subjektiven Beschwerden bzw. klinischen Befunden gesehen."}

Der sich rasch verändernde Kenntnisstand im Bereich „Umwelt und Gesundheit" und die Notwendigkeit zur interdisziplinären Zusammenarbeit waren sicherlich auch die Gründe dafür, dass im 1994 publizierten gesundheitspolitischen Programm der deutschen Ärzteschaft die Einrichtung elektronischer Kommunikationssysteme als Voraussetzung für eine kompetente umweltmedizinische Betreuung festgeschrieben wurde. Da sich in Deutschland nicht nur spezielle Umweltmediziner mit Fragen aus dem Bereich „Umwelt und Gesundheit" beschäftigen, wird für die weitere Betrachtungsweise in diesem Beitrag der Terminus der EH-Professionals (environmental health professionals) benutzt. Diese Bezeichnung steht auch im internationalen Einklang, da in keinem anderen europäischen Land eine ,umweltmedizinische" Zusatzbezeichnung oder ein (Fach)Arzt für Umweltmedizin analog der deutschen Weiterbildungsordnung existiert.

\section{Elektronische Netzwerke für EH-Professionals in Europa}

Die aus den Erfahrungen des Arbeitsalltags im Bereich „Umwelt und Gesundheit" in der Bundesrepublik resultieren-

\footnotetext{
U. Kaiser

Robert Koch-Institut, Postfach 650280 13302 Berlin
} 


\section{U.Kaiser}

Construction and improvement of a European information and communication platform for the area ,Environment and Health"

\section{Summary}

The area „Environment and Health" is characterized by interdisciplinary features and, consequently, an intense need for communication. In addition it has to be considered that sometimes there are difficulties in terms of information search. A comparison of national problem solving strategies concerning electronic network activities within this area led to the development project for a European information and communication platform. By this electronic platform the international exchange of experiences, coordination of activities, harmonization efforts and an optimization of qualification possibilities for environmental health professionals will become easier.

\section{Keywords}

Environmental health - Electronic network . European information- and communication platform de Einschätzung, dass einfach zu handhabende, überall nutzbare und vor allen Dingen überschaubare Wege der Informationsbeschaffung und der Kommunikation benötigt werden, führte 1993 zur Planung eines eigenen computergestützten Informations- und Kommunikationsnetzes zwischen den EH-Professionals.

\section{„Benötigt werden einfach zu handhabende und überschaubare Wege der Informationsbeschaffung und Kommunikation."}

In Zusammenarbeit zwischen der Dokumentations- und Informationsstelle für Umweltfragen (DISU) in Osnabrück und dem Robert Koch-Institut (RKI) in Berlin wurde das „Umweltmedizinische Informationsforum UmInfo" unter aktiver Mitgestaltung durch seine Nutzer entwickelt. Der Schwerpunkt dieses Online-Systems mit grafischer Benutzeroberfläche (Abb. 1) liegt in der Umweltmedizin, der Allergologie und in verwandten medizinischen Fachgebieten und kombiniert ein öffentliches Informationsangebot mit dem Übergang zum Intranet der EH-Professionals.
Das System bietet Zugriff auf validierte Daten und Fakten (Volltextdokumente), offene und berufsgruppeninterne Diskussionsebenen, interdisziplinären Erfahrungsaustausch und Übergänge $\mathrm{zu}$ weiteren Kooperationspartnern. Neben zahlreichen themenbezogenen „elektronischen Konferenzen” gibt es zugriffsgeschützte Bereiche für spezielle Nutzergruppen (Kinderärzte, öffentlicher Gesundheitsdienst etc.). Bei Bedarf können problemlos weitere geschützte Bereiche eingerichtet und von den Interessenten selbstständig moderiert werden. Neben der Intention, mit einer elektronischen Vernetzung den vertikalen Informationsfluss (Richtund Grenzwerte, Verordnungen, etc.) zu verbessern, ist eine besondere Stärke des Systems in der horizontalen Vernetzung und damit beispielsweise im Zugriff auf den ,kollektiv gewachsenen Erfahrungsschatz" zu sehen.

Optimalen Komfort und hohe Sicherheit bietet das System beim Zugang mit der kostenlosen Software FirstClass ${ }^{\circledR}$. Der Zugang kann durch telefonische Direkteinwahl über $13 \mathrm{Re}$ gionalknoten erfolgen oder über das Internet-Protokoll „TCP-IP”. Das System kann aber auch direkt mit einem ,WebBrowser" (Netscape, Internet-Explorer) benutzt werden. Als Kosten fallen für

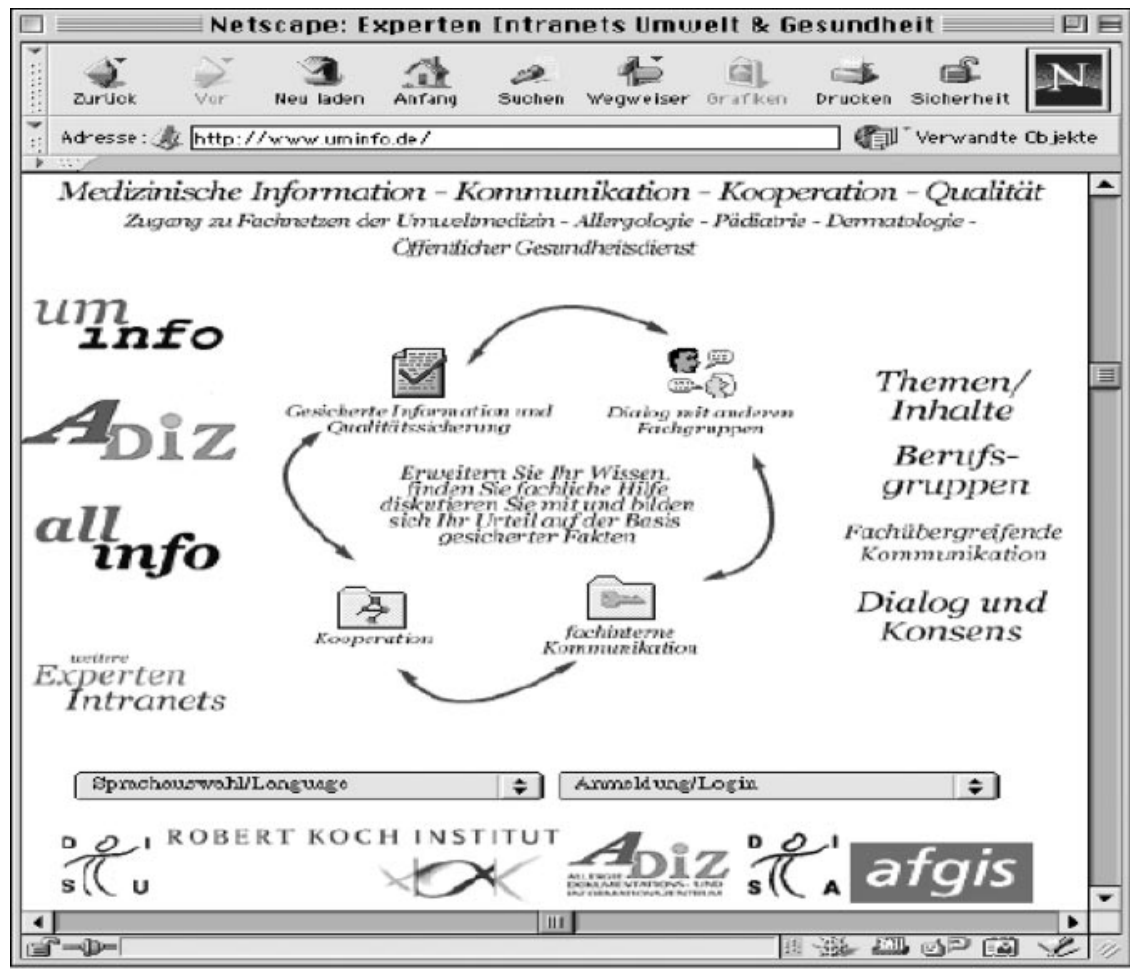

Abb. $1 \Delta$ Die UmInfo-Homepage im Internet 


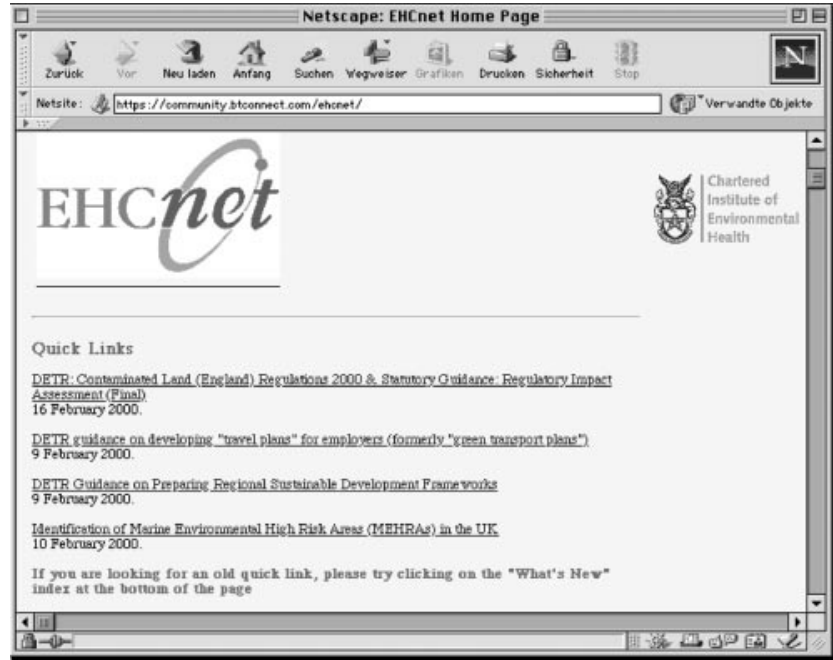

Abb. $2<$ Ausschnitt aus dem Eingangsbildschirm des EHCnet den Nutzer nur die Telefonkosten bzw. Internetprovidergebühren an.

Eine etwas andere Entwicklung zeigt das EHCnet des Chartered Institute of Environmental Health (CIEH) in England (Abb. 2). Ende 1980 ist das EHCnet aus einem elektronischen Warnsystem für akute Gesundheitsgefahren entstanden und hat sich zu einem Netzwerk für den schnellen und vertraulichen Informations- und Erfahrungsaustausch staatlicher und freiberuflicher EH-Professionals entwickelt. Für den nicht unerheblichen Jahresbeitrag von etwa 2000 DM erhalten die Nutzer Zugang zu einem ,qualitätskontrollierten Internet". Die Mitarbeiter des CIEH überprüfen im ,freien Internet” erreichbare Informationsquellen bezüglich Qualität und Relevanz für EH-Professionals. Nur positiv bewertete URLs (Uniform Resource Locator = Internetadressen wie z.B. www.who.dk etc.) können über dieses System angesteuert werden. Da in England zahlreiche elektronisch erreichbare „Web-Sites” offizieller Stellen mit Volltextdokumenten verfügbar sind, ergibt sich aus dieser Vorauswahl ein sehr zeiteffizient nutzbarer Informationspool.

\section{„Der EHCnet-Ansatz gewinnt an Bedeutung, da Arbeitgeber den freien Internetzugang inzwischen kritisch beurteilen."}

Der im „freien Internet” im allgemeinen notwendige hohe Zeitaufwand für das Selektieren zwischen wertlosen und relevanten Informationen für $\mathrm{EH}$ Professionals entfällt hier zum Preis einer Beschneidung der „Informationsfreiheit". Da inzwischen auch in der Bundesrepublik die Beobachtung gemacht wird, dass Arbeitgeber immer häufiger den „,freien Internetzugang” für alle Mitarbeiter aufgrund sinnloser „Surfaktivitäten” kritisch im Vergleich zum Nutzen thematisieren, gewinnt der EHCnet-Ansatz an Bedeutung. Neben der primären Intention des Systems, den „,top-down-Informationsfluss” zu verbessern, wird auch der horizontale Informationsfluss durch E-Mail-Funktionalität und zahlreiche Mailinglisten zumindest ansatzweise gefördert und unterstützt.

In Frankreich wurde das RESE (Réseau d'Échanges en Santé Environnementale) 1996 vom Gesundheitsministerium initiiert, um den Informationsfluss zur Basis zu verbessern (Abb. 3). Das Netzwerk ist nur staatlichen EH-Professionals auf zentraler und lokaler Ebene und Kooperationspartnern des Gesundheitsministeriums zugänglich. Die Nutzer haben neben E-mail, automatischen Meldungen über neue Gesetzestexte und sonstige Regelwerke Zugriff auf eine Fülle von Volltextdokumenten $\mathrm{zu}$ Arbeitsmethoden, Qualitätssicherung, Fachinformationen, hilfreiche Internetlinks, Bürgerinformationsbroschüren etc. auf dem Gebiet des umweltbezogenen Gesundheitsschutzes. Der Informationsfluss wird primär vertikal verbessert. Eine horizontale Kommunikationsverbesserung im Sinne eines ,kollektiven Erfahrungsaustausches" beispielsweise durch Nutzung/Einbindung gemeinsamer Diskussionsforen etc. wird in diesem Netzwerk kaum unterstützt. Das System arbeitet kostenlos für die Nutzer.

\section{"Eine Verbesserung der Kommunikation zwischen EH-Professionals durch Nutzung von elektronischen Kommunikationssystemen wäre wünschenswert."}

Neben UmInfo, EHCnet und RESE gibt es in der europäischen Region der WHO (52 Nationen) keine weiteren zugriffsgeschützten Expertennetze für den Bereich „Umwelt und Gesundheit”. Einzelne Länder haben aber eine Verbesserung der Kommunikationen zwischen

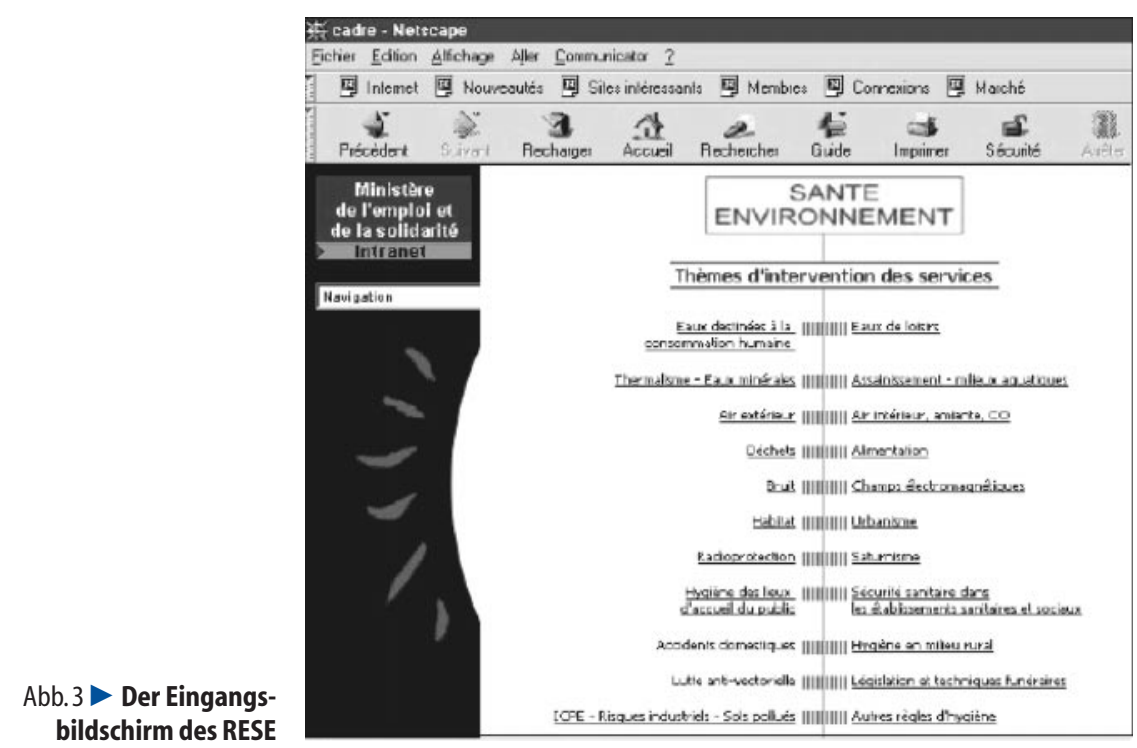


den EH-Professionals durch nahezu flächendeckend vorhandene E-Mail-Funktionalität (z.B. Finnland) erreicht. Seitens der WHO/EURO wurde im Rahmen der Entwicklung des „Environmental Health Action Plan for Europe" (EHAPE) die Bedeutung der Nutzung elektronischer Informations- und Kommunikationssysteme auf diesem Gebiet unterstrichen und deren Auf- bzw. Ausbau im Rahmen der „National Environmental Health Action Plans" (NEHAPs) gefordert.

1997 eröffnete die Weltgesundheitsorganisation ein neues WHO-Zentrum (collaborating centre) am Robert KochInstitut in Berlin. Das in Zusammenarbeit mit dem Bundesministerium für Gesundheit konzipierte und finanzierte Projekt ist auf internationale Zusammenarbeit ausgerichtet und verfügt hierfür über je eine Fachkraft für den englischen und russischen Sprachraum. Die im Titel geführte Bezeichnung „WHO - Collaborating Centre for Information and Communication on Environmental Health" unterstreicht den steigenden Bedarf nach koordiniertem Informationsaustausch und professionellem Informationsmanagement auf dem Gebiet „Umwelt und Gesundheit” und stellt ein weiteres Bindeglied zwischen nationalen und internationalen Aktivitäten im Rahmen des NEHAPProzesses dar.

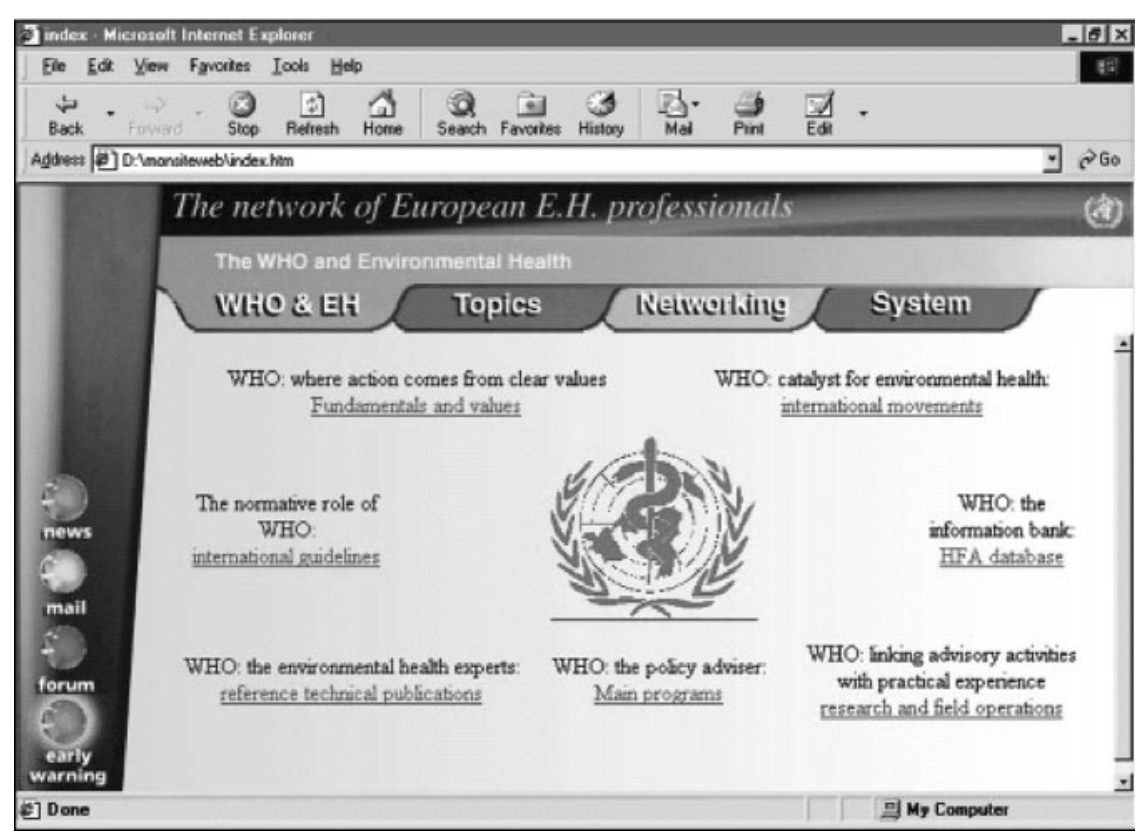

Abb. 4 Ausschnitt aus der Demo-Präsentation der geplanten europäischen Kommunikationsplattform
In Absprache mit der WHO/EURO wurde vom WHO-CC am RKI in Zusammenarbeit mit den Netzwerkbetreiben von EHCnet, RESE und UmInfo auf der Umwelt- und Gesundheitsministerkonferenz in London (1999) eine Demo-Präsentation „Towards a European Network in Environmental Health" vorgeführt (Abb. 4).

Die Vorarbeiten zu diesem Projekt wurden auf einem vom WHO-CC organisierten Workshop in Berlin/Potsdam (Januar 1999) erarbeitet. Dort hatten zwölf Vetreter aus sechs Nationen die grundlegenden Anforderungen an ein europäisches Netz thematisiert. Trotz der internationalen Sprachplattform „Englisch” wurde am Konzept der nationalen Netze in der jeweiligen Landessprache festgehalten, da nach wie vor die Notwendigkeit einer intensiven kollegialen Zusammenarbeit unter Einbeziehung nationaler/regionaler Besonderheiten dominiert. In zunehmendem Maße werden aber auch durch globale Umweltprobleme, Vordringen des EURechts auf nationaler Ebene oder durch stärkere Berücksichtigung von Standards (Richt- oder Grenzwerte etc.) anderer Nationen bei der eigenen (nationalen) Risikokommunikation europäische Vernetzungsaspekte angeführt. Aufgrund der vorhandenen Sprachbarrieren wurde die Notwendigkeit profes- sioneller Übersetzungsdienste in einem europäischen Netz festgeschrieben. Es wurde ein schrittweises Vorgehen vereinbart. Auf der Basis einer „Pilot-Plattform" sollen erste Erfahrungen durch die Länder mit bereits vorhandenen elektronischen Zugriffsmöglichkeiten gesammelt werden. Für Länder ohne Netzwerkarchitektur soll eine entsprechende Anleitung zum Aufbau vernetzter Strukturen im Bereich „Umwelt und Gesundheit" auf nationaler Ebene erstellt werden.

\section{"Globale Umweltprobleme, das EU-Recht und stärkere Berücksichtigung von Standards anderer Nationen erfordern eine europäische Vernetzung."}

Die sich auf der Ministerkonferenz in London durch die Präsentation ergebenden Gespräche mit Vertretern zahlreicher Nationen bestätigten diese Vorgehensweise. In Zusammenarbeit mit der WHO/EURO wurde seitens des RKI an der Konkretisierung des Projekts gearbeitet. In der Europäischen Kommission wurde ein geeigneter Ansprechpartner für eine Anschubfinanzierung gefunden. Ein vom RKI Ende 1999 gestellter Förderantrag wurde Anfang 2000 von Brüssel genehmigt und mit entsprechenden finanziellen Mitteln ausgerüstet. Das Management für das Projekt „Improving information and communication management in the field of pollution-related diseases by strengthening the contribution of $\mathrm{EH}$ professionals through electronic networks" wurde im Bereich Umweltmedizin des RKI angesiedelt und hat in $\mathrm{Zu}$ sammenarbeit mit dem WHO-CC mit der technischen und inhaltlichen Ausgestaltung der europäischen Kommunikationsplattform „EHCom-Platform” begonnen. Aktiv beteiligt an der Entwicklungsarbeit sind ebenfalls die Netzwerkpartner der Pilotstudie EHCnet UmInfo - RESE. Als „Beitrittsländer” der Phase 1 (2000) sind Finnland, Bulgarien und Tschechien weitere beteiligte Nationen.

An das Projekt wurden folgende komplexe Aufgaben gestellt, um zukünftig in den europäischen Nationen für den Bereich „Umwelt und Gesundheit” 
angemessene Handlungsoptionen und Präventionsmöglichkeiten zu gewährleisten:

D Verbesserung des Hintergrundwissens über umweltbezogene Erkrankungen

D Unterstützung der Validierung von Studien/Daten zu umweltbezogenen Erkrankungen (z.B. Trends in der Prävalenz) mit Hilfe der bestehenden Netzwerke; Identifizierung prioritärer Problembereiche und Ableitung adäquater Maßnahmen; Identifizierung von Wissenslücken und daraus abzuleitender Forschungsbedarf

D Verbesserung des Managements umweltbezogener Erkrankungen

- Angleichung der „Beobachtungsparameter" in den verschiedenen Nationen zum besseren „Pooling” von Daten; Erfahrungsaustausch über Maßnahmen und Handlungsmöglichkeiten; Erfahrungsaustausch über Risikowahrnehmung und Risikokommunikation

D Qualifizierung der Strukturen im Bereich „Umwelt und Gesundheit” Entwicklung von Aufbauszenarien für die elektronische Vernetzung von EH-Professionals in Ländern mit bisher fehlender Netzwerkarchitektur; Entwicklung abgestimmter Vorgehensweisen und Präventionsstrategien
Im Detail sollen sich zahlreiche Vorteile für die Nutzer der Komunikationsplattform ergeben:

\section{Serviceleistungen für die Teilnehmer}

D Volltextdokumentenarchiv (WHO, EC Dokumente, relevante wissenschaftliche Literatur),

D Gesetzestexte, Vorlagen für Erhebungsinstrumente etc.,

D Zugriff auf die aufzubauende Datenbank „Who's Who in Environmental Health" zur besseren Transparenz und zum Auffinden relevanter Ansprechpartner,

D elektronische Austauschkonferenzen „aus der Praxis - für die Praxis” zur gegenseitigen Hilfe,

D elektronische Querverweise auf bewertete und empfohlene Internetbereiche (validierte Hyperlinks),

D Newsletter, Veranstaltungskalender, Stellenmarkt.

\section{Unterstützung beim Auf- und Aus- bau nationaler Netzwerkstrukturen}

D Handbuch mit generellen Anforderungen und technischen Grundlagen,

D Erfahrungsaustausch mit anderen Ländern bezüglich Anlaufschwierigkeiten,

D Hilfe bei der Organisation entsprechender Schulungsmaßnahmen.

\section{Zusammenarbeit mit Organisatio- nen und internationalen Programmen}

D Aufbau geordneter Informationsund Kommunikationswege mit WHO, IPCS, EEA, IFEH, etc.

D Aufbau eines „Frühwarnsystems” zur besseren Identifizierung sich neu abzeichnender Problemfelder,

D frühzeitige Koordination abzustimmender Maßnahmen,

D Verbesserung der Zugriffsmöglichkeiten auf relevante Datenbanken,

D Kurzberichte und Übersichten über neue nationale und internationale Regelwerke,

D Unterstützung von Standardisierungsbemühungen zur Verbesserungs des Datenaustauschs,

D Zugriff auf ein multilinguales Glossar „Environmental health”.

\section{Optimierung der Zusammenarbeit zwischen der nationalen und internationalen Netzwerkarchitektur}

D Schaffung von „Gateways” zur internationalen Plattform,

D Unterstützung beim Aufbau notwendiger Übersetzungskapazitäten,

D dauerhafte finanzielle Absicherung der EHCom-Plattform und Sicherstellung der inhaltlichen Arbeit.

Eine am Jahresende vorzulegende Analyse der Aufbauphase soll die exakte Planungsgrundlage für den schrittweisen Ausbau liefern und somit den Anschluss weiterer Nationen konkretisieren. Entsprechende Projektverlängerunganträge bei der Europäischen Kommission sollen die Netzwerkentwicklung bis 2003 absichern. 